\title{
ASOCIACIONISMO EN RED PARA LA INTEGRACIÓN SOCIODIGITAL EN ANDALUCÍA ${ }^{1}$
}

\section{NETWORK ASSOCIATIONS FOR SOCIODIGITAL INTEGRATION IN ANDALUSIA}

\author{
Gustavo Contreras Cabrera \\ Universidad de Sevilla \\ gcontreras@us.es
}

Recibido: marzo, 2012.

Versión final aceptada: enero, 2013.

PALABRAS CLAVE: red, brecha digital, servicios públicos, territorio.

KEYWORDS: network, digital divide, public services, territory.

\section{RESUMEN}

Los centros de acceso público a Internet llegan a conformar redes en la distribución geográfica donde actúan, prestando servicios en torno a objetivos comunes. Pero también, es posible distinguir estructuras en red relacionadas con otros aspectos organizativos más complejos, destacando el grado de madurez existente en la consolidación del servicio o la propia consideración de éste último como un hecho limitado a la desaparición de las causas que lo han originado. En Andalucía existe la red de centros Guadalinfo orientada a la inclusión social en materia digital que ha sido analizada en su diseño y funcionamiento a través de este trabajo de investigación.

Entre las principales conclusiones obtenidas destacan las importantes carencias exploratorias y explicativas de los resultados conseguidos y de los impactos demostrables en la población beneficiaria, así como los vacíos de implantación e importantes déficits de asistencia pública a la ciudadanía, independientemente de su dinámica o riqueza socioeconómica o del tamaño poblacional de los núcleos urbanos.

\footnotetext{
${ }^{1}$ Los contenidos y desarrollos de este artículo son derivados de la investigación sobre la planificación e impronta socioterritorial de esta red llevada a cabo a entre 2009 y 2012 mediante la Tesis Doctoral "Las políticas de Inclusión en la sociedad de la información en Andalucía: Planificación e Impronta socioterritorial de la red de centros de acceso público a Internet (Programa Guadalinfo)", así como del proyecto de investigación "Estrategia de innovación empresarial. Comportamientos territoriales" del Plan Nacional I+D+i del Ministerio de Economía y Competitividad (2012-2014). REF: CO 2011-26125.
}

ISSN: 0212-8594 ISSN-e: 2340-2776 № DOI: http://dx.doi.org/10.12795/rea.2012.i29.05 REA 29 (2012):105-126

http://www.publius.us.es/estudios_andaluces 


\section{ABSTRACT}

The centers of public Internet access make up networks in the geographical distribution where they operate, giving services around common goals. Yet, it is also possible to distinguish between network structures related to other more complex organizational aspects. This distinction emphasizes the degree of maturity in the consolidation of the service or the own consideration of that service as a limited fact to the disappearance of the causes that originated it. In Andalusia, there exists the network of Guadalinfo whose objective is the inclusion in digital subject, which has been analyzed in its design and operation through this research work.

Among the main conclusions, it can be highlighted the considerable exploratory and explanatory gaps of the achieved results and the demonstrable impacts on the target population, as well as the implementation gaps and significant deficits of public assistance to the citizens, regardless of wealth or socioeconomic dynamics or the population size of the urban centers. 


\section{INTRODUCCIÓN.}

Los centros de acceso público a Internet llegan a conformar redes en la distribución geográfica donde actúan, prestando servicios que pivotan en torno a objetivos comunes. Pero también, es posible distinguir estructuras en red relacionadas con otros aspectos organizativos más complejos, destacando el grado de madurez existente en la consolidación del servicio o la propia consideración de éste último como un hecho limitado a la desaparición de las causas que lo han originado.

Este tipo de centros surgió en Andalucía fruto de iniciativas, en su mayoría espontáneas, de agentes o instituciones privadas y públicas, generalmente sin ánimo de lucro, entre las que se encontraban las Administraciones más cercanas a la ciudadanía, como la Administración Local. En este último caso, cabe destacar la acción de ayuntamientos con mayor capacidad en cuanto a recursos (humanos, técnicos y económicos). Dicho fenómeno se fue extendiendo por el territorio andaluz como expresión innovadora y complementaria de la oferta de servicios a la ciudadanía impulsados desde centros municipales asociados, entre otros, a la cultura (Bibliotecas Públicas o Casas de la Cultura), a la juventud (Casas de la Juventud o Centros de Actividades Extraescolares), así como a los movimientos asociativos/ vecinales (Centros Cívicos o Centros de Dinamización Sociocultural de Barrios).

Tras el éxito de estas experiencias en los ámbitos territoriales donde se fueron desarrollando, la Administración Autonómica de Andalucía inicia la implantación de una red homogénea de centros de acceso público a Internet a través del denominado Programa Regional de Acciones innovadoras - PRAl- Guadalinfo (2002-2004). Dicha estructura superó esa primera etapa experimental, impulsándose en los años siguientes en aquellos municipios andaluces con población igual o inferior a 10.000 habitantes, con una clara vocación correctora de la exclusión sociodigital en municipios donde se presumía una mayor falta de recursos públicos locales para garantizar el acceso de la población a Internet. Posteriormente, se han venido produciendo ampliaciones en la extensión de la experiencia Guadalinfo, según el perfil de los Municipios beneficiarios y la necesidad de corregir nuevos desequilibrios socioterritoriales (en municipios con población entre 10.000 y 20.000 habitantes, barriadas urbanas desfavorecidas o comunidades andaluzas en el exterior), hasta llegar a la realidad dinamizadora y territorial de la actual red andaluza de inclusión.

\section{HIPÓTESIS, OBJETIVOS Y MARCO TEÓRICO.}

La investigación del proceso de diseño estratégico y de la puesta en funcionamiento de la red de centros Guadalinfo ha permitido profundizar en las circunstancias estratégicas de su implantación en el territorio, su nivel de cobertura o las expectativas ciudadanas en torno a posibles mejoras en su funcionamiento. En dicho sentido, fueron fijadas inicialmente varias hipótesis de trabajo y otros tantos objetivos

ISSN: 0212-8594 ISSN-e: 2340-2776 № DOI: http://dx.doi.org/10.12795/rea.2012.i29.05 
de estudio, cuya formulación ha marcado el hilo conductor de la investigación, hasta llegar a las conclusiones finales que de manera sintética serán expuestas más delante.

a) Una primera hipótesis señaló la eventualidad de que la experiencia Guadalinfo respondiera a un diseño estratégico coherente con las políticas y estrategias impulsadas en el contexto político-administrativo y territorial en el que se integra la Comunidad Autónoma de Andalucía.

b) En segundo lugar se consideraba la posibilidad de que el proceso de implantación y desarrollo de la red Guadalinfo obedeciera a unos planteamientos iniciales de diseño ya superados, no garantizadores de la equidad socioterritorial en materia de inclusión sociodigital.

c) Así mismo, se intuía la oportunidad de mejorar el modelo andaluz de inclusión sociodigital, mediante la ampliación de la capacidad de acceso de la población objetivo a los centros de acceso público a Internet, su participación en el diseño estratégico de iniciativas y resolución de expectativas ciudadanas.

Como referente al desarrollo del estudio y verificación de estos supuestos iniciales se propuso una cadena secuencial de objetivos a alcanzar. A partir de un objetivo principal de avance en el conocimiento de las políticas de integración y dinamización sociodigital existentes en Andalucía, proyectadas a través de la red Guadalinfo, se fijaron las siguientes metas específicas:

1. Interpretación del proceso estratégico-territorial configurador de esta red.

2. Verificación de su equidad en el alcance socioterritorial como resultado de la implantación realizada.

3. Identificación de sus impactos y potencialidades.

A su vez, parte del marco teórico tomado como referencia para esta investigación giró en torno a tres cuestiones esenciales referidas a las políticas de alfabetización y dinamización digital, a los centros de acceso público a Internet y a las redes institucionales de centros vinculadas a un modelo de servicio público de acción positiva.

\subsection{LAS POLÍTICAS DE ALFABETIZACIÓN Y DINAMIZACIÓN DIGITAL.}

La alfabetización y dinamización digital representan una parte esencial en las políticas de acción positiva para la reducción de desequilibrios socioterritoriales relacionados con la plena integración individual o colectiva en la sociedad informacional. 
Aunque existen importantes esfuerzos públicos y privados en torno al acceso efectivo de la sociedad a las tecnologías de la información y la comunicación (TIC), la mera disponibilidad de equipos o de tecnologías de información y comunicación no se pueden considerar viables para garantizar dicha integración. La brecha digital tiene su origen en causas diversas y complejas que requieren formas de intervención integradoras y persistentes, relacionadas con el desarrollo de competencias ciudadanas. Por ello, la alfabetización y dinamización digital representan un enfoque capacitador relacionado con la formación ciudadana en la adquisición de habilidades y conocimientos próximos a los de un individuo cívicamente competente (Selwyn, N., 2004):

"Aquel capaz de conocer, hacer y tener una actitud en un contexto determinado de actuación en los planos políticos, sociales y económicos".

Algunos autores (García, I., 2005; Castells, M. 2006) destacan tres componentes en la acción positiva de la inclusión digital, referidos a la corrección de posibles déficits en la disponibilidad o acceso ciudadano al hardware y a otros equipos relacionados con las TIC. La corrección de posibles déficits en la disponibilidad o acceso ciudadano al hardware y a otros equipos relacionados con las TIC; el acceso efectivo a Internet como garantía de equidad; y el desarrollo de capacidades ciudadanas necesarias para el uso y aprovechamiento de estas tecnologías.

La acción positiva constituye así una respuesta válida a un desequilibrio digital de carácter multidimensional, aunque no llega a otorgar a ninguna de las potenciales contribuciones TIC un protagonismo especial en el conjunto de posibilidades definidas (Van Winden, W., 2003) (fig.1).

Figura 1: Dimensiones de la Inclusión como acción positiva.

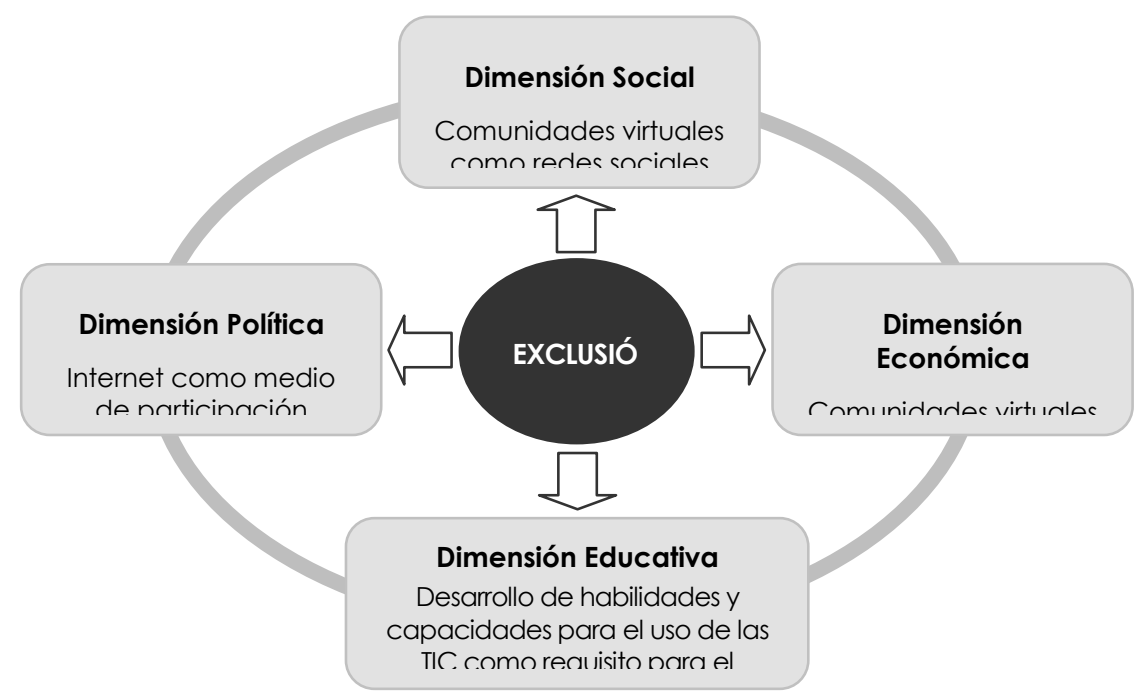

Fuente: Elaboración propia, a partir de Essays on urban ICT policies (W. Van Windem, 2003).

ISSN: 0212-8594 ISSN-e: 2340-2776 № DOI: http://dx.doi.org/10.12795/rea.2012.i29.05

REA 29 (2012):105-126

http://www.publius.us.es/estudios_andaluces 
A su vez, resulta oportuno atender a la dinámica experimentada por la alfabetización y dinamización digital, desde su comprensión como simple fórmula de acercamiento y familiarización con las innovaciones tecnológicas en el campo de la información y las comunicaciones, pasando por la instrucción ciudadana en el uso de diversas aplicativos asociados a estas tecnologías, para llegar a una concepción mucho más amplia e integradora, la sitúa estas prácticas como partes esenciales del propio sistema educativo existente.

Conviene mencionar el carácter transversal de la instrucción digital que otorgan diversos autores a esa acción positiva. De hecho, es defendida la idoneidad de incorporarla a todo el conjunto de actividades formativas, independientemente de la consecución que se produzca de habilidades específicas (Casado, R., 2007). En ese sentido, surgen posturas que admiten la existencia de diferentes etapas relacionadas con el proceso de integración de las TIC en la educación (Boza, A. y otros, 2010):

- Etapa de toma de conciencia, acerca de las ventajas y efectos beneficiosos aportados a la educación por las TIC.

- Etapa integradora, caracterizada por la aportación de recursos técnicos y humanos cualificados.

- Etapa transformadora, que responde a la plena implantación de las TIC en las acciones didácticas, así como en las tareas organizativas de los diferentes centros educativos.

\subsection{LOS CENTROS DE ACCESO PÚBLICO A INTERNET.}

El concepto de centro de acceso público a Internet reúne vínculos especialmente directos con el carácter de acción positiva que la alfabetización-dinamización digital posee (Hecht, L., 1998). Dentro del contexto dinamizador y/o educativo que representan estos centros, es preciso considerar su vinculación a procesos de educación gratuita y no formal (Casado, R., 2007), siendo admitida la existencia de diferentes posturas en torno a su cometido:

- Libre acceso a las TIC e Internet: Se trata de poner este equipamiento a disposición de la ciudadanía para contrarrestar posibles dificultades o carencias tecnológicas.

- Ofrecer acciones formativas (sobre el uso y aprovechamiento de las TIC o sobre determinados temas apoyados en el uso de las mismas como recurso). Este planteamiento se acerca más a la capacidad de las TIC para prestar apoyo a la enseñanza en general, así como a la posibilidad de reducir la brecha digital mediante su conocimiento y aprendizaje. 
- Involucrar a la sociedad en general en el acercamiento a las TIC dentro de un proceso integrador de inmersión en la sociedad de la información (alfabetización-dinamización digital), superando posibles deficiencias de conocimiento en su manejo e impulso a la participación ciudadana.

Otros investigadores sostienen la posibilidad de encuadrar estos centros según el tipo de población beneficiaria. Para ellos, puede existir una orientación generalista, sin llegar a hacer distinciones entre las personas objeto del servicio, o bien atender a determinados grupos de población, como desempleados, discapacitados, inmigrantes, jóvenes o minorías étnicas, localizando los centros en puntos geográficos donde existe una mayor proporción de población objetivo y enfocando sus estrategias de trabajo hacia unas necesidades concretas.

Finalmente, se han podido constatar investigaciones y modelos teóricos acerca de la capacidad de los centros para acceder, difundir o crear contenidos, identificándose estos últimos con la información y el conocimiento, así como la forma de presentarlos e interpretarlos, junto a la especificidad de la acción positiva para reducir la brecha digital (Castells, M., 2006).

Los centros que estimulan el uso o producción de contenidos pueden generar un impacto importante en los individuos o en la comunidad objetivo, como el refuerzo de ciertos valores, el reconocimiento del sentido de la colaboración o la resolución de problemas aparentemente complejos. Para ello, es preciso considerar diversas opciones estratégicas y seleccionar la más apropiada.

\subsection{LAS REDES INSTITUCIONALES DE CENTROS DE ACCESO PÚBLICO A INTERNET.}

Las redes de centros de acceso público a Internet pueden generarse en base a estrategias vinculadas a políticas de acción positiva para la integración digital. En este caso, se puede hablar de fórmulas de intervención, públicas o privadas, que parten de una cierta planificación en la puesta en marcha de acciones y en la asignación de recursos materiales, económicos y humanos (Castells, M., 2006), llegando incluso a captar e implementar estrategias innovadoras de marcada utilidad (Ferrero, F. y De la Cuesta, G. A., 2007).

La distribución espacial de estos centros responde en muchos casos a un diagnóstico previo que identifica problemas a solventar o necesidades a cubrir, desarrollando a su vez unos objetivos y líneas de actuación acordes a la población objetivo y a los recursos disponibles, además de fijar desde un punto de vista organizativo un modelo de servicio sujeto a una doble condición, según su estructura organizativa y según su contenido y población beneficiaria, que fundamenta a posibilidad de construir una red prestadora de servicios de carácter universal o personal (Tarroja, À. y Camagni, R., 2006).

ISSN: 0212-8594 ISSN-e: 2340-2776 № DOI: http://dx.doi.org/10.12795/rea.2012.i29.05 


\section{METODOLOGÍA APLICADA.}

La metodología seguida en esta investigación se sustenta en el análisis de las políticas públicas de integración en la Sociedad de la Información aplicadas mediante el desarrollo de la red Guadalinfo en el conjunto del territorio de la Comunidad Autónoma de Andalucía, a escala regional y también mediante el estudio de casos concretos de comarcas andaluzas con diferentes niveles de dinamismo socioeconómico y de inequidad sociodigital.

Estos enfoques toman como premisa general la existencia de restricciones que dificultan o imposibilitan efectivamente el flujo de la ciudadanía andaluza hacia la sociedad de la información, dando lugar a la existencia de una brecha entre personas y territorios que se intenta corregir por medio de acciones de integración promovidas por Administraciones públicas (por ejemplo, la Autonómica de Andalucía), (fig. 2).

Figura 2. Incidencia de la inclusión sociodigital.

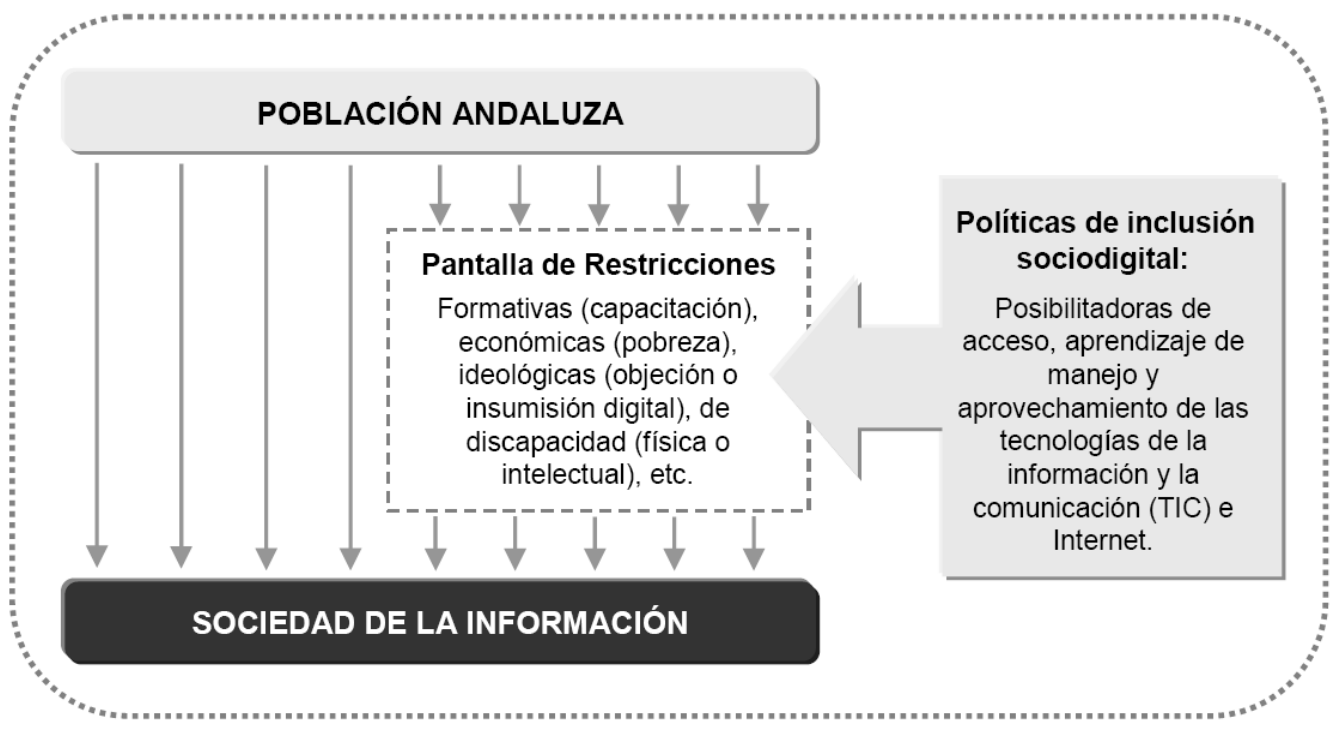

Fuente: Elaboración propia.

Así mismo, la investigación se ha basado en un triple modelo de análisis con cobertura en el diseño estratégico de inclusión sociodigital emprendido desde la Administración Autonómica Andaluza, el alcance socioterritorial de su estrategia Guadalinfo, y el impacto y potencialidades derivados de la misma:

El análisis del diseño estratégico parte de la puesta en marcha de un examen pormenorizado de la figura emblemática que rige la acción integradora de la Administración Autonómica en Andalucía: el Plan Estratégico Guadalinfo (2009-2012). Este análisis se apoya en el enfoque del Marco Lógico, vinculado estrechamente a la

ISSN: 0212-8594 ISSN-e: 2340-2776 № DOI: http://dx.doi.org/10.12795/rea.2012.i29.05 
evaluación conceptual o de diseño de políticas, planes o estrategias basados en un modelo de acción por objetivos ${ }^{2}$.

Por su parte, el análisis del alcance socioterritorial de la estrategia Guadalinfo fija su acción en el cálculo de la impronta que la red ha tenido sobre la población y el territorio, de acuerdo con tres bloques generales de evaluación relativos al perfil identificativo de la población objetivo, a la lógica de la correspondencia socioterritorial, y a la adecuación del modelo topológico y funcional de red. Estos bloques se desarrollan en niveles específicos para construir una imagen inequívoca de la citada impronta socioterritorial.

Finalmente, el análisis del impacto y potencialidades de la estrategia Guadalinfo resulta especialmente vinculado a la verificación del planteamiento hipotético tercero propuesto inicialmente. Este componente de análisis se encuentra dividido en tres grandes bloques operativos de análisis que han sido examinados con suficiente detalle para la interpretación de su incidencia y potencialidades en el servicio a la ciudadanía andaluza.

Avanzando en las consideraciones realizadas acerca del empleo del territorio global de la Comunidad Autónoma de Andalucía, así como de determinadas comarcas y municipios incluidos en las mismas, conviene indicar que el análisis expuesto ha contado con la importante acción diferenciadora de las diversas variables examinadas (como la disponibilidad de recursos TIC e Internet o el grado de capacitación en su uso y aprovechamiento por parte de la población residente, o la adecuación de la política de comunicación de los centros de la red Guadalinfo), y con la atención a la convergencia entre los valores resultantes de los territorios seleccionados. Ello, ha permitido incluso el estudio comparativo entre los llamados territorios ricos o dinámicos, bajo un punto de vista del desarrollo socioeconómico, y otros territorios que la propia Administración Autonómica ha calificado como "a revitalizar".

También ha sido contemplada la existencia de municipios con población superior a 20.000 habitantes dotados de centro de atención sociodigital en determinadas barriadas consideradas como desfavorecidas, por existir colectivos ciudadanos en situación real de exclusión social (minorías étnicas, población extranjera, bolsas de pobreza o altos niveles de desempleo, etc.) en estrecha relación con la exclusión digital. Sin embargo, la falta de información a este nivel de detalle ha hecho que sean analizadas a escala municipal, integradas en las comarcas ya mencionadas.

\footnotetext{
${ }^{2}$ Dado que la planificación Guadalinfo ya era una realidad al iniciarse esta investigación, se consideró fundamental comprobar su grado de racionalidad y de coherencia, analizándose los elementos justificativos de su necesidad y estrategia, concretamente su relevancia (validez de su diagnóstico de situación), su pertinencia (centrada en la calidad de la definición de sus objetivos general y de desarrollo), así como su concordancia o alineamiento interno (línea de causalidad entre diagnóstico, objetivos, estrategias y acciones operativas) y externo (planificación o estrategias del entorno).
}

ISSN: 0212-8594 ISSN-e: 2340-2776 № DOI: http://dx.doi.org/10.12795/rea.2012.i29.05 
Así pues, la distinción entre territorios se ha realizado a varios niveles, como los relacionados con la disponibilidad de centros de acceso público a Internet, determinando la existencia de vacíos dotacionales (totales o parciales), el tamaño poblacional de los municipios, o el nivel de dinamismo y desarrollo socioeconómico de éstos.

Esta última precisión toma como referencia dos comarcalizaciones diferentes, cuya validez ha sido ampliamente reconocida desde distintos foros de investigación e institucionales, cuyo interés se proyecta en la comprobación de la vigencia de los planteamientos iniciales de implantación de esta red.

Las comarcas a revitalizar (fig. 3) han sido extraídas de la zonificación territorial del Programa de Desarrollo Rural Sostenible (PDRS) 2010-2014 implementado en esta Comunidad Autónoma. El PDRS surge al amparo de la Ley 45/2007, de 13 de diciembre, para el desarrollo sostenible del medio rural. Entre sus acciones impulsadas para la revitalización de estos espacios, prevé la reducción de desequilibrios en materia de tecnologías de la información y la comunicación. Por otra parte, los ámbitos comarcales más dinámicos se han seleccionado a partir de la clasificación territorial, llevada a cabo por Cano García (2001 y 2002).

Figura 3. Reparto tipológico de comarcas a analizar.

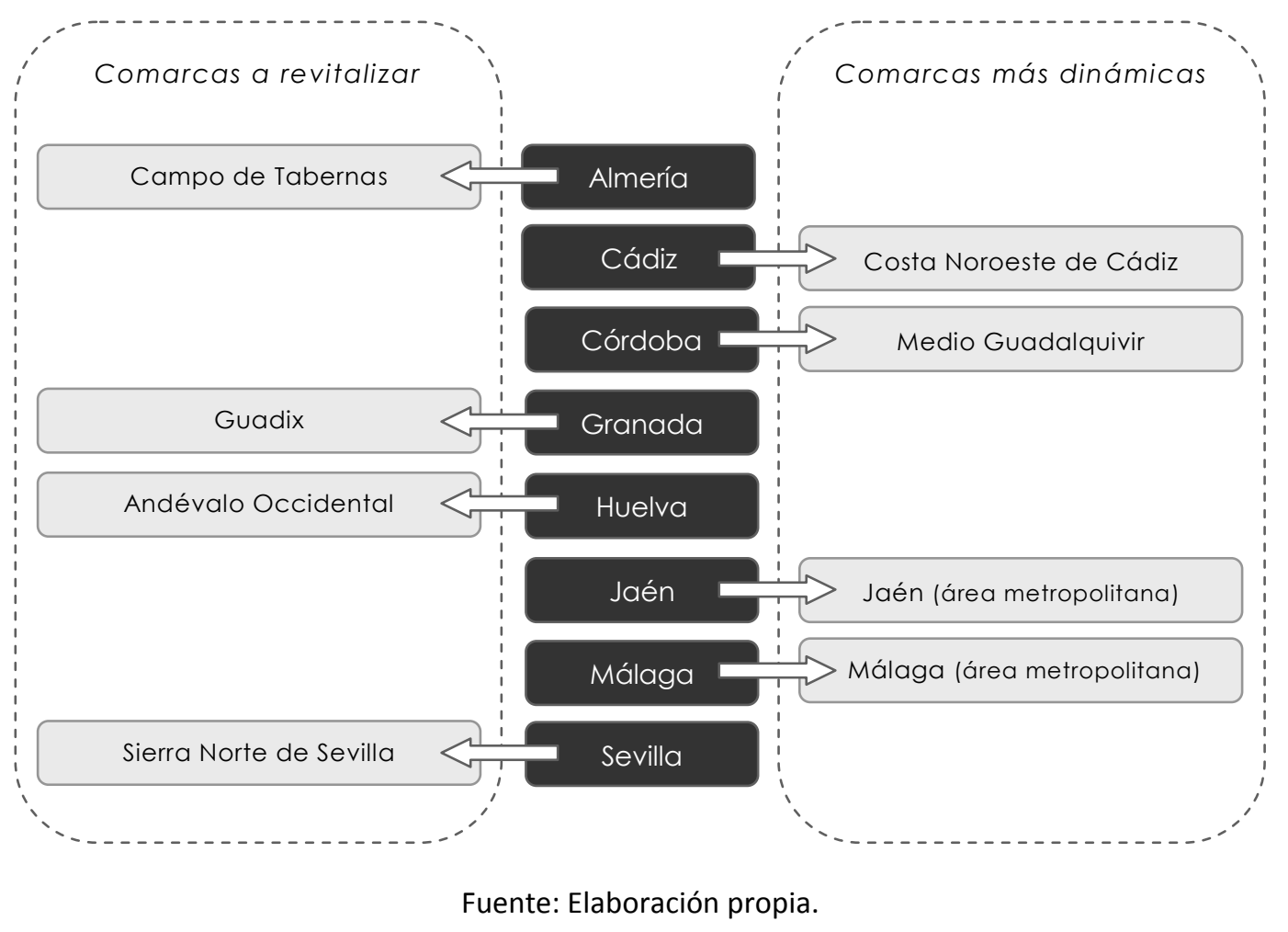

ISSN: 0212-8594 ISSN-e: 2340-2776 № DOI: http://dx.doi.org/10.12795/rea.2012.i29.05 REA 29 (2012):105-126

http://www.publius.us.es/estudios_andaluces 


\section{DESARROLLO ANALÍTICO Y RESULTADOS DESTACADOS.}

El análisis de la experiencia abierta con el diseño y despliegue de la red Guadalinfo en Andalucía ha constituido un reto especialmente atractivo en el marco de acción de esta investigación, todo ello siguiendo el mencionado triple enfoque señalado anteriormente.

a) Del diseño estratégico de las acciones de inclusión sociodigital.

b) Del alcance socioterritorial de la experiencia Guadalinfo.

c) De los impactos y potencialidades de la red.

Desde esta multiperspectiva de análisis y sus correspondientes interpretaciones se ha buscado dar respuesta a las cuestiones fundamentales del estudio, relacionadas con el alcance de las metas marcadas para este trabajo o la verificación de sus hipótesis iniciales. Los resultados más destacables son expuestos a continuación.

\subsection{DISEÑO ESTRATÉGICO DE LAS ACCIONES DE INCLUSIÓN SOCIODIGITAL.}

Del análisis del diseño estratégico de la red Guadalinfo se obtienen resultados de especial trascendencia para un mejor conocimiento de su estructura organizativa y gestora, así como de las condiciones que rigen su implementación en el territorio a través del Plan Estratégico Guadalinfo:

El mapa de actores contemplados en su gestión y funcionamiento posee una importante fuerza estructurante y organizativa, aunque en él se precia una asignación de roles que parece no responder adecuadamente al modelo de fórmula organizativa que requiere esta extensa red de centros, por el desproporcionado peso decisorio y rector de la Administración Autonómica de Andalucía y de las Diputaciones provinciales frente al papel sumamente subordinado de los actores locales.

Por otra parte, ha sido analizada la participación de entidades colaboradoras en barriadas desfavorecidas y en ámbitos referidos a Comunidades Andaluzas en el Exterior $^{3}$, cuyas características no garantizan una estabilidad en el servicio a la población beneficiaria.

\footnotetext{
3 Existen diversas entidades que gestionan el funcionamiento de centros de la Red Guadalinfo en barriadas desfavorecidas (asociaciones vecinales y otras entidades dedicadas a labores específicas como el tratamiento de la drogodependencia o la integración de minorías étnicas e inmigrantes). Por su parte, las Comunidades Andaluzas en el Exterior, actúan como entidades de apoyo a los andaluces emigrantes en el exterior de Andalucía en el mantenimiento de la identidad sociocultural andaluza o en la asistencia e información a nuevos emigrantes.
}

ISSN: 0212-8594 ISSN-e: 2340-2776 № DOI: http://dx.doi.org/10.12795/rea.2012.i29.05

REA 29 (2012):105-126

http://www.publius.us.es/estudios_andaluces 
Se ha podido comprobar la insuficiencia del aprovechamiento de las posibilidades de mejora del seguimiento y coordinación entre agentes gestores de las entidades locales y agentes dinamizadores. Así mismo, es posible encajar la participación de las entidades locales en el Consorcio gestor y la sustitución de incentivos por convenios de colaboración.

La siguiente figura (fig. 4) muestra el mapa de actores construido tras analizar las principales competencias y características de los agentes e instituciones que intervienen en esta red de centros:

Figura 4. Cuadro de agentes del Plan Estratégico Guadalinfo 2009-2012.

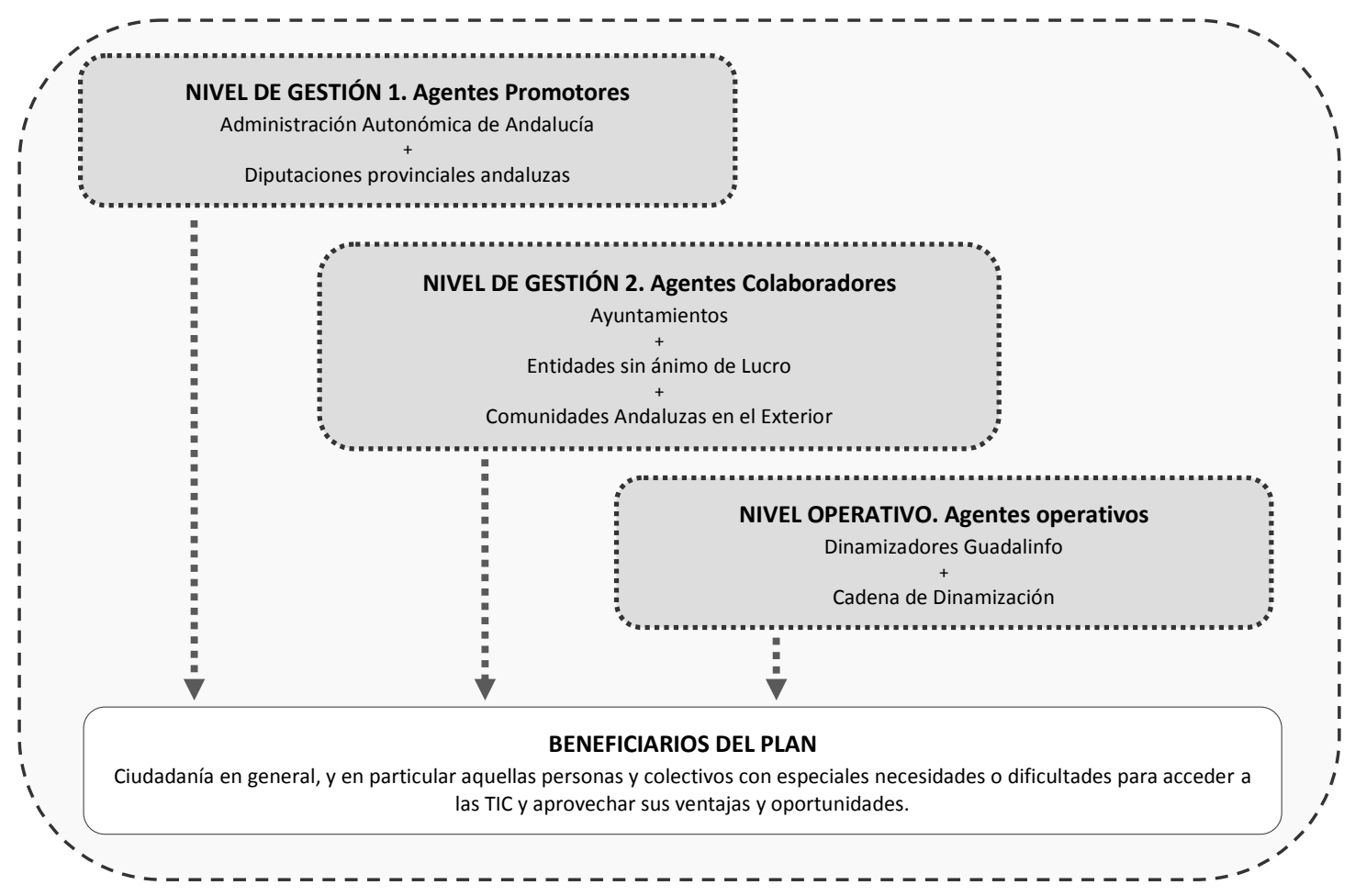

Fuente: Elaboración propia.

Entrando directamente en la propia planificación estratégica de la red Guadalinfo, se aprecia que la racionalidad carece de relevancia en su diseño, más dirigida por las inercias de su trayectoria de trabajo (desde el año 2002) que por un diagnóstico formal y fundamentado. Además, el análisis de su pertinencia refleja una importante debilidad motivada por la falta de claridad y de concreción en algunos de los compromisos asumidos y en sus planteamientos estratégicos.

En cuanto a la coherencia resultante, en su faceta externa presenta un alineamiento adecuado con los intereses y retos del contexto en que se desarrolla. Por contra, existe un importante salto argumental entre sus objetivos y las estrategias de actuación diseñadas. Incluso es llamativa su falta de respuesta a un escenario de partida con necesidades de transformación, dada su evidente indefinición, y la

ISSN: 0212-8594 ISSN-e: 2340-2776 № DOI: http://dx.doi.org/10.12795/rea.2012.i29.05 
carencia identificativa de los principales factores del entorno y sus posibles interferencias.

Una de las citadas incoherencias internas del Plan es consecuencia de una labor de acoplamiento de dos estructuras de objetivos que dan como resultado una figura más compleja y definitiva (fig. 5).

Figura 5. Acoplamiento de objetivos del Plan Estratégico Guadalinfo.

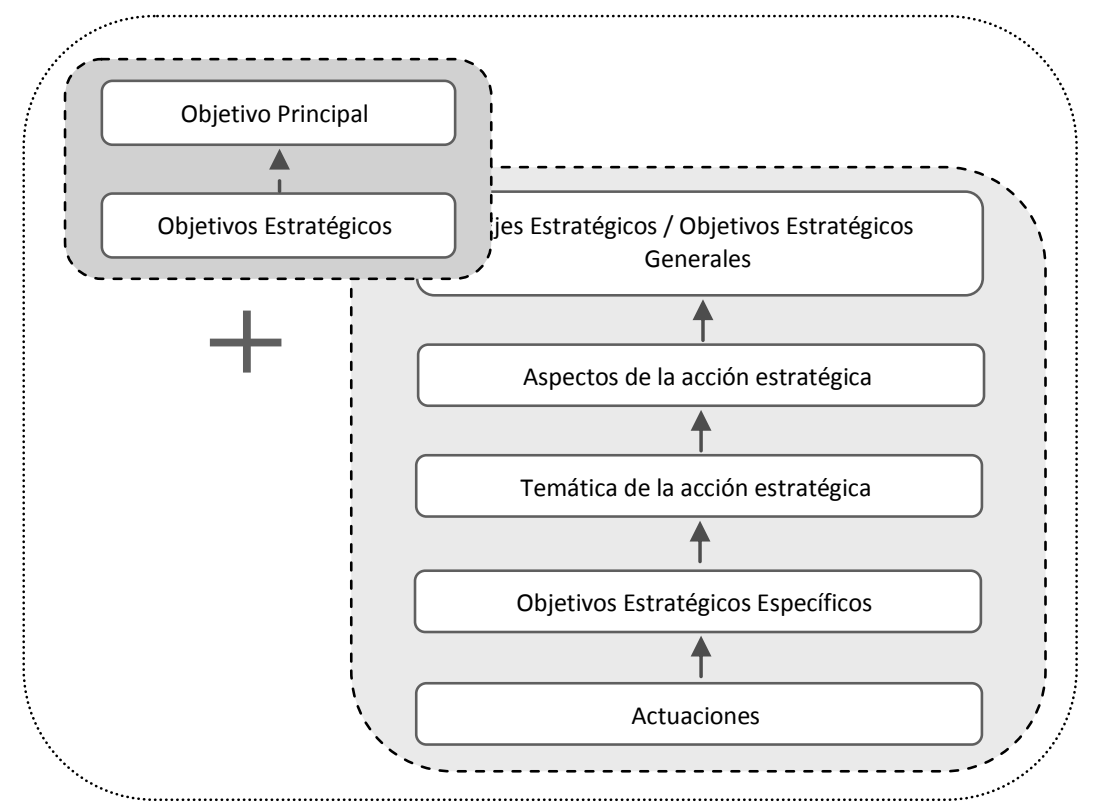

Fuente: Elaboración propia, a partir del Plan Estratégico Guadalinfo 2009-2012.

En este contexto, resulta excesiva la consideración de los ejes estratégicos (Ciudadanía y Gestión) como objetivos estratégicos generales, dada la ausencia de fijación de metas, y al responder más bien a una división funcional u operativa de las estrategias diseñadas. Esta forzada situación produce un estrechamiento inusual en la estructura piramidal resultante que se hubiese resuelto sin problemas configurando el nivel de objetivos estratégicos de forma que quedase reflejada esta doble orientación de enfoque a la ciudadanía y a la gestión de esta red.

\subsection{ALCANCE SOCIOTERRITORIAL DE LA EXPERIENCIA GUADALINFO.}

El perfil identificativo de la población objetivo de la acción Guadalinfo viene marcado por la existencia de rasgos característicos del riesgo de exclusión social. En este sentido, se han detectado importantes carencias de acceso y manejo de las TIC e Internet vinculadas a otras insuficiencias (esencialmente económicas y de conocimientos para su uso), todas ellas en directa proporción con sus elevadas aspiraciones de integración sociodigital. 
Por otra parte, ha resultado alarmante el elevado nivel de desconocimiento de la población entrevistada acerca de la existencia de la red Guadalinfo, así como de la presencia de algún centro de esta red en la localidad de residencia de las personas entrevistadas. De este modo, se evidencian fallos de información/comunicación acerca de los servicios, ventajas y potencialidades de la realidad Guadalinfo (gráf.1).

Gráfico 1. Conocimiento de la experiencia Guadalinfo y de la existencia de centro en el municipio de los entrevistados.

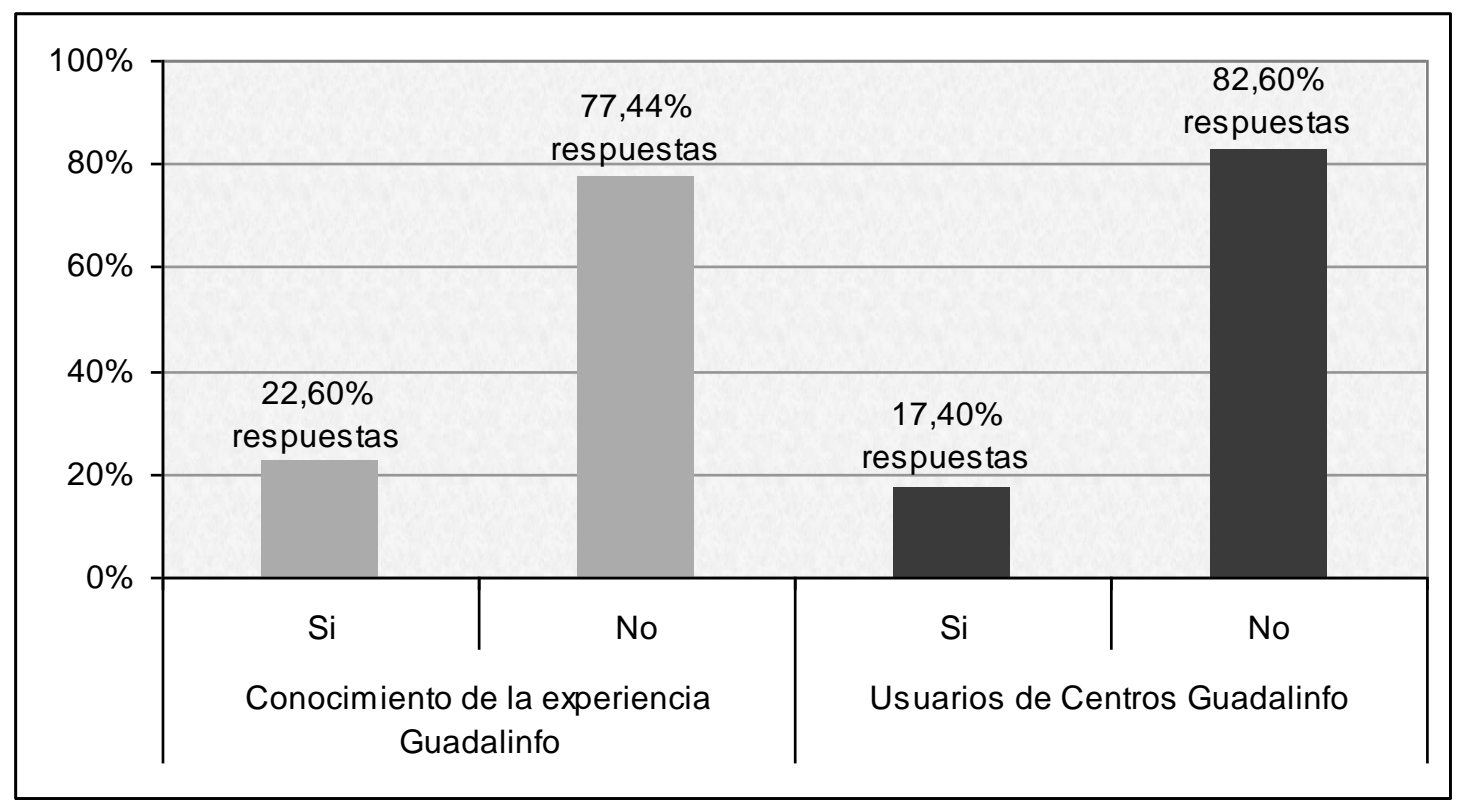

Fuente: Elaboración propia.

Otro resultado del análisis realizado ha sido la constatación de la pérdida de la lógica inicial en la correspondencia socioterritorial de la red, debido a sus diferencias y vacíos de cobertura, así como de los servicios prestados a los habitantes de cada territorio objeto de estudio.

El Análisis de la proporcionalidad existente entre la población objetivo y la oferta real de centros de la red Guadalinfo se ha realizado mediante la elaboración de cinco ratio ${ }^{4}$ o indicadores de beneficiarios por centro implantado y de los vacíos socioterritoriales

\footnotetext{
4 1.-Ratio de población que no dispone de equipo informático en casa/no centros vinculados a la red Guadalinfo en cada comarca; 2.-Ratio de población sin acceso a internet en casa/ no centros vinculados a la red Guadalinfo en cada comarca; 3.-Ratio de población con necesidades altas de integración digital/ $\mathrm{n}$ ㅇ centros vinculados a la red Guadalinfo en cada comarca; 4.-Ratio de usuarios oficiales registrados/ n으 centros vinculados a la red Guadalinfo en cada comarca.5.- Ratio de población con nivel bajo y nulo/muy bajo en el manejo TIC e internet / no centros vinculados a la red Guadalinfo en cada comarca.
}

ISSN: 0212-8594 ISSN-e: 2340-2776 № DOI: http://dx.doi.org/10.12795/rea.2012.i29.05

REA 29 (2012):105-126

http://www.publius.us.es/estudios_andaluces 
de esta red, detectándose grandes diferencias intercomarcales, de acuerdo con el poblamiento y el reparto de estos centros (gráf.2).

Los territorios con mayor desarrollo socioeconómico han presentado ratios de carencias ciudadanas más abultados en torno a las TIC e Internet por cada centro existente. De igual modo sucede con las capacidades de acceso y manejo TIC e Internet, además de la actitud mostrada hacia una plena integración en la sociedad de la información. Por otra parte, se ha apreciado una diferencia general considerable entre las personas residentes en los ámbitos territoriales sondeados con necesidades de acceso, manejo y cualificación digital, respecto de los usuarios registrados en los centros pertenecientes a esta red de integración, circunstancia especialmente destacada en las comarcas más desarrolladas.

Gráfico 2. Ratios comarcales resultantes del análisis de correspondencia entre la población objetivo y los centros existentes.

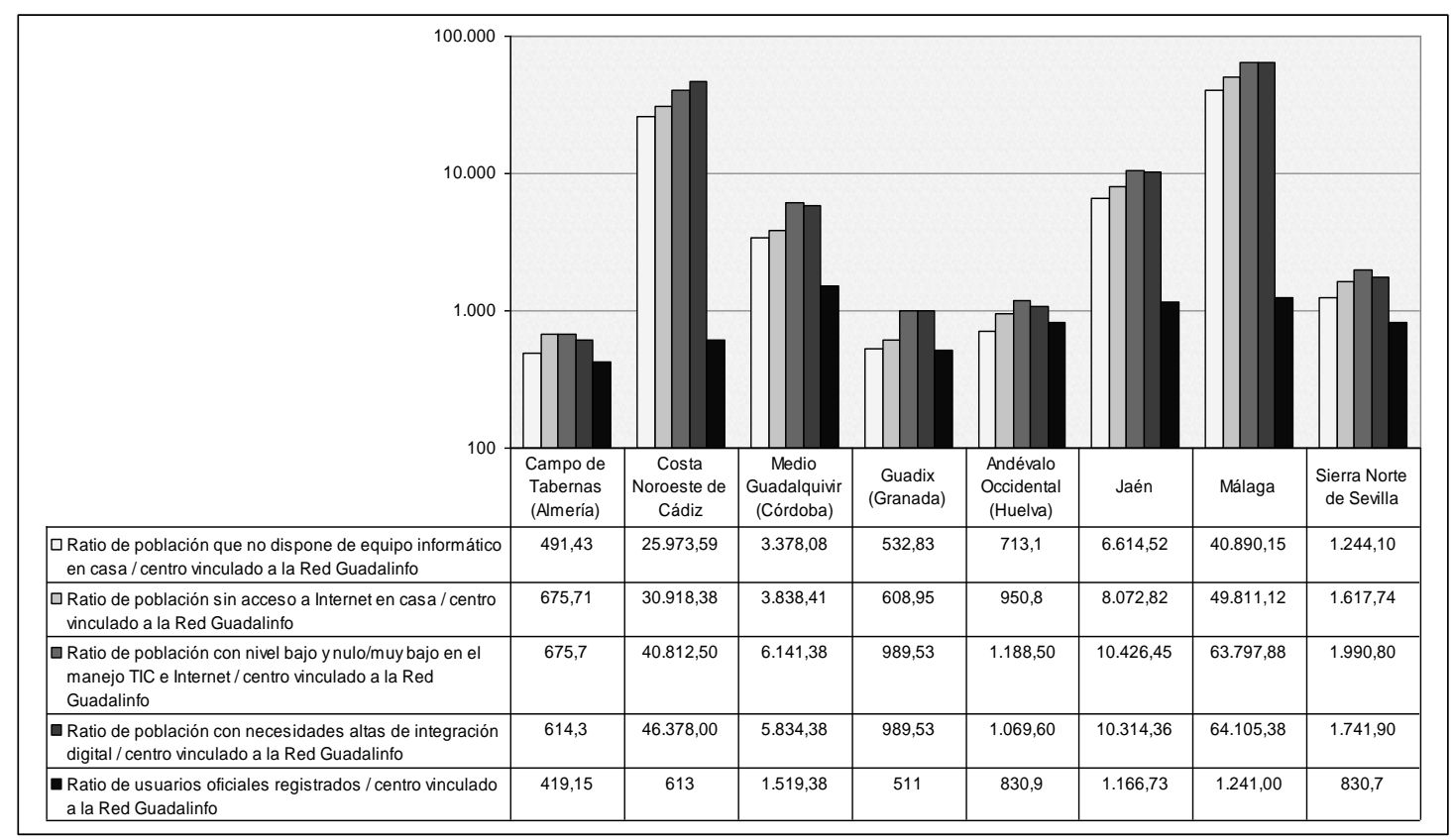

Fuente: Elaboración propia.

Para finalizar este bloque analítico, un resultado de especial valor ha sido la verificación del $20,14 \%$ de la población andaluza (casi 1.700 .000 habitantes) residente en 37 municipios carentes de centros de la red Guadalinfo (mapa 1). Situación que se agrava al añadir vacíos territoriales parciales, de acuerdo con la distribución impulsada de centros de acceso público a Internet en determinadas barriadas desfavorecidas de otros municipios. Este reparto no ofrece cobertura a la totalidad de la población residente donde se encuentran implantados, debido a su vinculación a proyectos orientados a determinados segmentos de población (como minorías étnicas, con problemas de drogadicción, inmigrantes, etc.). Los mayores vacíos se han registrado

ISSN: 0212-8594 ISSN-e: 2340-2776 № DOI: http://dx.doi.org/10.12795/rea.2012.i29.05 
en Málaga y Coste Noroeste de Cádiz, aunque destacan globalmente los cuatro territorios señalados por su mayor desarrollo socioeconómico.

Mapa 1. Municipios sin cobertura o con cobertura parcial de la red Guadalinfo.

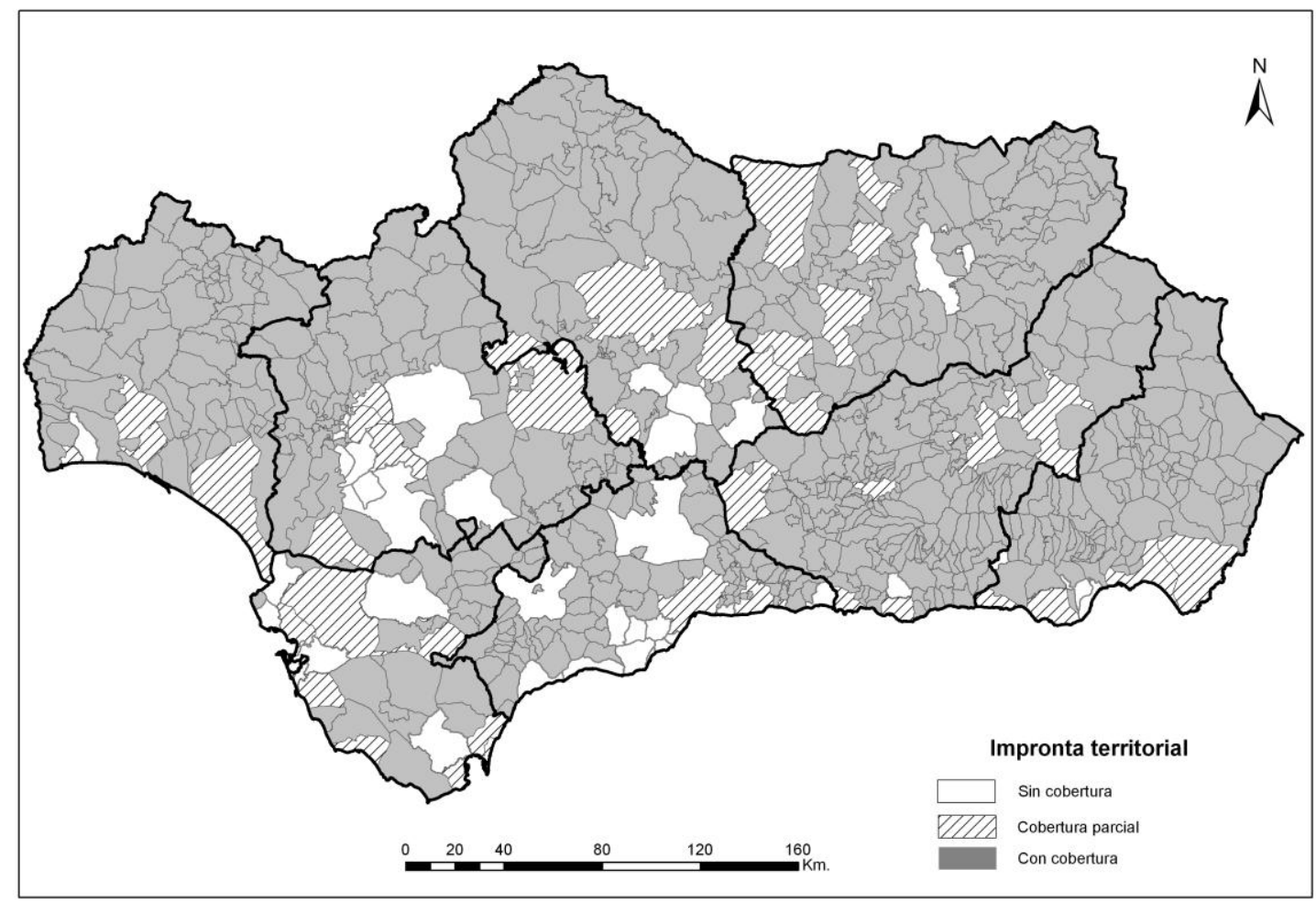

Fuente: Elaboración propia.

\subsection{IMPACTOS Y POTENCIALIDADES DE LA RED.}

Los impactos y potencialidades de la realidad Guadalinfo en Andalucía cierran el proceso de análisis impulsado en esta investigación, mostrando interesantes resultados.

Tanto en su globalidad territorial andaluza, como a nivel de cada comarca analizada, las preferencias (cualificación personal y de aprovechamiento o manejo de los recursos TIC disponibles) presentan un asombroso paralelismo con los impactos percibidos, determinándose la existencia de una relación evidente de causa-efecto entre las experiencias obtenidas a través de los centros de la red Guadalinfo y las preferencias mostradas.

La percepción del grado de beneficios concretos que los centros de esta red han proporcionado a los entrevistados han sido "altos" y "muy altos" en su mayoría, aunque con una repercusión limitada en el conjunto de usuarios potenciales. Esta

ISSN: 0212-8594 ISSN-e: 2340-2776 № DOI: http://dx.doi.org/10.12795/rea.2012.i29.05 
debilidad se explica por la deficiente política de difusión de objetivos, temáticas, acciones y resultados de la acción Guadalinfo (gráfs. 3 y 4).

Gráfico 3. Distribución comparativa de los niveles de aprovechamiento de la oferta de los centros y su efectividad difusora percibida.

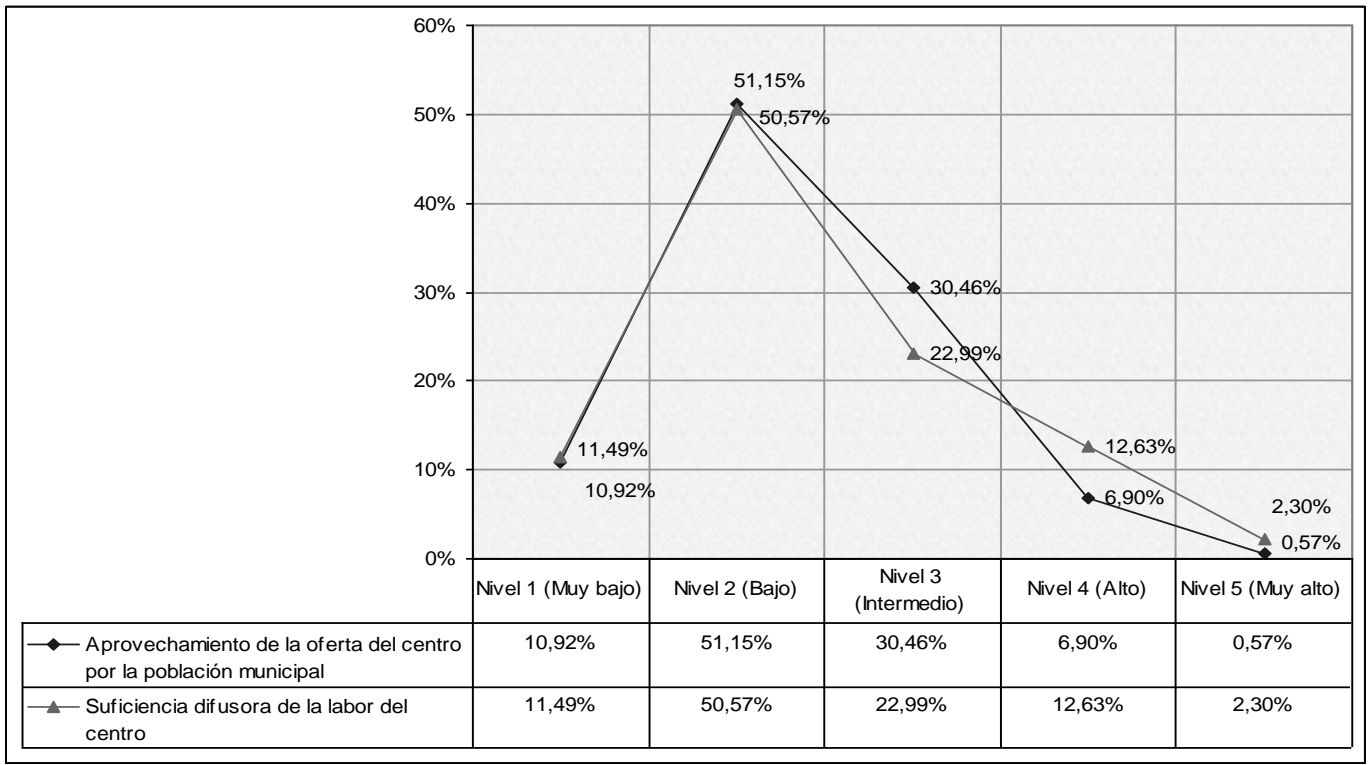

Fuente: Elaboración propia

Gráfico 4. Distribución comarcal de los impactos globales más beneficiosos para los usuarios entrevistados.

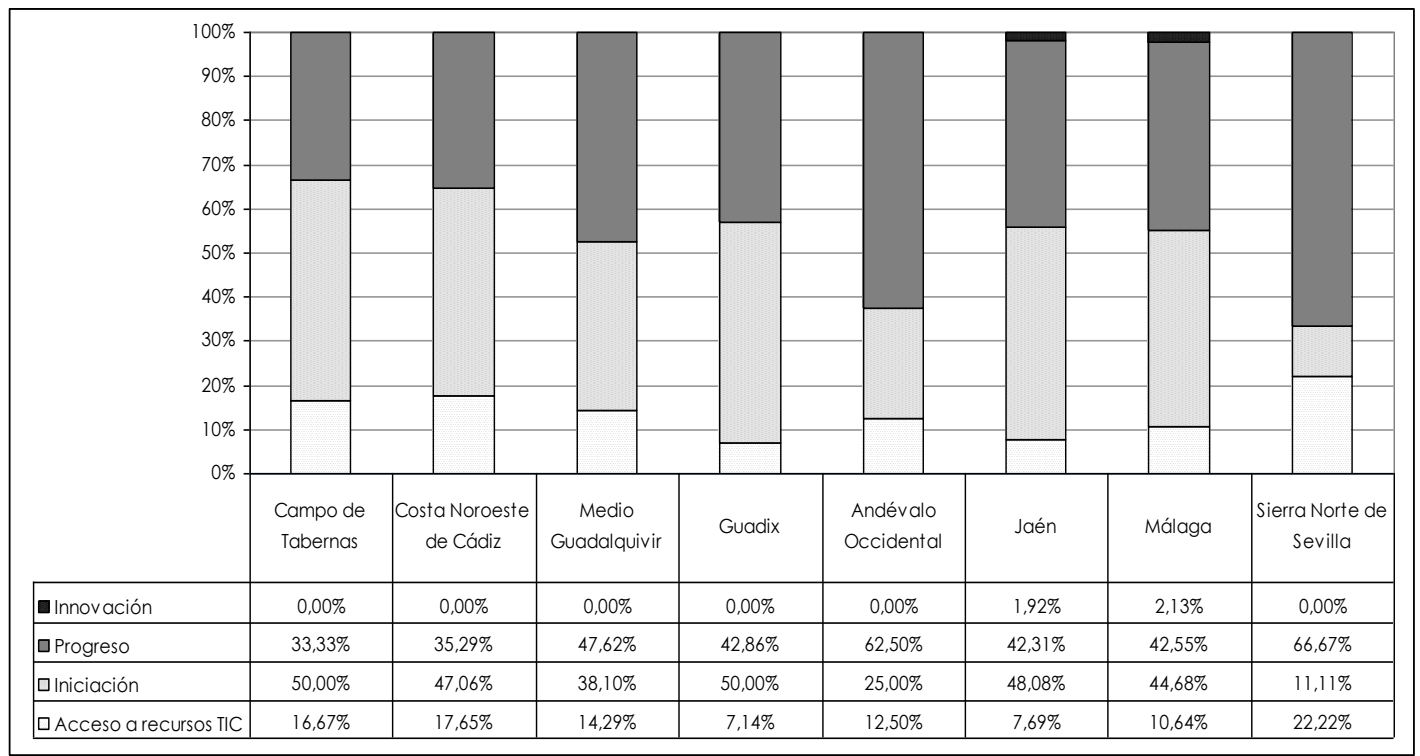

Fuente: Elaboración propia.

ISSN: 0212-8594 ISSN-e: 2340-2776 № DOI: http://dx.doi.org/10.12795/rea.2012.i29.05

REA 29 (2012):105-126

http://www.publius.us.es/estudios_andaluces 
Los mayores beneficios globales coinciden con la iniciación y el progreso en el uso y aprovechamiento de las tecnologías asociadas a la sociedad de la información, visualizándose con nitidez un predominio del impacto formativo sobre el acceso a los recursos tecnológicos.

La visualización de la cooperación entre centros de la red y la Administración Local ha sido confirmada por la población beneficiaria, aunque con un significativo grupo de personas incapaces de distinguirla con detalle. Entre las áreas o departamentos municipales más vinculadas al proceso de integración sociodigital en cooperación con los centros Guadalinfo destacan "Asuntos sociales", "Educación" y "Promoción Económica/Empleo", en clara diferencia con otras opciones.

Las potencialidades de la red son percibidas en forma de mejoras concretas relacionadas con las aportaciones de especialistas en temáticas de especial interés formativo y de conocimiento para sus usuarios, una política de información más eficaz y la cobertura de determinadas carencias de equipamiento en los centros. Así mismo, se aprecia la posibilidad de ampliar la red de centros de la iniciativa Guadalinfo al conjunto de municipios que carecen en la actualidad de ese servicio, y un elevado nivel de fidelidad de los usuarios hacia sus respectivos centros de referencia.

Otros resultados destacables en las expectativas ciudadanas de la red coinciden con la universalización de su servicio, entendido como una prestación de carácter básico, estable y en crecimiento, aunque no queda clara la finalidad del mismo, al existir prácticamente un empate técnico entre las perspectivas de un servicio complementario al bienestar y la cualificación ciudadana, y las de exclusividad hacia el acceso y aprendizaje en el uso de las tecnologías de la información y las comunicaciones.

\section{CONCLUSIONES GENERALES.}

Una constante aspiración vinculada a esta investigación ha residido en la idea de profundizar desde un punto de vista teórico en el fenómeno de la inequidad sociodigital surgida en paralelo al desarrollo de la sociedad de la información, así como en las fórmulas correctoras impulsadas en forma de redes territoriales de contención. Si bien se ha detectado un cuerpo teórico e interpretativo extenso y profundo respecto del concepto sociedad de la información, así como de su consecuencia negativa más destacada, -la brecha digital,- apenas se han identificado estudios centrados en las políticas públicas de integración sociodigital, incluidos sus estrategias y métodos más adecuados para atajar las inequidades surgidas.

Es en el diseño de pautas e instrumentos válidos y su puesta en práctica, donde se han apreciado los mayores vacíos exploratorios y explicativos sobre los resultados conseguidos y los impactos demostrables en la población beneficiaria. Tanto en el ámbito de la Unión Europea, como en el marco del Estado Español y sus Comunidades 
Autónomas (en este caso, Andalucía), se han detectado políticas de inclusión más bien vinculadas a un procedimiento de ensayo y aprendizaje (prueba-error) que ha permitido avanzar en su eficacia, eficiencia y equidad de forma gradual y con diferentes niveles de incertidumbre.

Al fijar la escala de estudio en la Comunidad Autónoma andaluza, se ha llevado a cabo un análisis múltiple de la realidad de estas políticas: en su diseño, en su impronta socioterritorial y en sus impactos, sinergias y expectativas de futuro, cuyos resultados han aclarado ciertamente el escenario de la inclusión sociodigital y sus principales carencias, principalmente metodológicas en la planificación y en la gestión.

Así mismo, el acercamiento realizado a lo que bien podría ser un índice de medición de la inequidad distributiva de los centros de acción integradora, -la ratio de cobertura territorial-, ha demostrado la existencia de vacíos de prestación o de importantes déficits de asistencia pública a la ciudadanía, independientemente de su dinámica o riqueza socioeconómica o del tamaño poblacional de los núcleos urbanos, con especial gravedad en municipios con mayor poblamiento. Las ciudades y municipios con población superior a los 20.000 habitantes concentran su cobertura de centros en barriadas o zonas consideradas como desfavorecidas, que responden a una realidad o caracterización de marginalidad social detectada paralela o desencadenante de la exclusión digital.

En estos últimos casos, no ha sido posible encontrar un cuerpo justificativo claro que diferencie o excluya de la prestación o servicio público a su población, en general, dadas las carencias evidentes de acceso y aprovechamiento de las TIC e Internet. Una prueba clara de esta deficiente política de implantación de la red Guadalinfo está en la falta de vigencia demostrada de algunos referentes paliativos de partida, que dieron como válido el criterio de localización preferente en zonas rurales aisladas o más desfavorecidas desde el punto de vista socioeconómico. Si bien, se ha registrado un aumento considerable de usuarios en las zonas con mayor grado de ruralidad, esta cuestión acentúa las diferencias existentes con otras zonas cuya cobertura es nula o parcial, desvirtuando la acción positiva emprendida.

En referencia al impacto de la implantación y funcionamiento de esta red sobre la población objetivo, se han detectado importantes beneficios, declarados por los propios beneficiarios sondeados, aunque constatándose igualmente aspectos a mejorar, en las prácticas divulgadoras de los servicios y ventajas asociadas a esta red de centros o en la recurrencia a la participación ciudadana en su diseño estratégico, actual y futuro.

Finalmente, la población beneficiaria percibe en esta red territorial diversas potencialidades a considerar por parte de los agentes gestores responsables de su funcionamiento y continuidad, vinculando su futuro a un modelo de servicio estable $y$ universal, dotado de cauces participativos más amplios en la toma de decisiones y en

ISSN: 0212-8594 ISSN-e: 2340-2776 № DOI: http://dx.doi.org/10.12795/rea.2012.i29.05 
la propia programación de actuaciones y contenidos acordes con las necesidades y aspiraciones de la sociedad andaluza.

Estas conclusiones globales confirman la validez de las hipótesis de partida formuladas en esta investigación, dada la coherencia de la red Guadalinfo en su diseño con el contexto estratégico externo, así como la falta de validez de unos criterios de implantación ya superados, o la posibilidad de avanzar en su gestión acometiendo mayores esfuerzos en materia de gobernanza y aprovechamiento de las expectativas ciudadanas. Igualmente, cabe confirmar el cumplimiento del propósito principal que ha guiado su implementación, al haber progresado en el conocimiento de los impulsos públicos en materia de integración sociodigital llevados a cabo en Andalucía. Esta meta ha sido alcanzada por medio de la interpretación del proceso estratégico y territorial de configuración de la red de centros Guadalinfo, comprobando el grado de equidad de su implantación, especialmente en lo referente a déficits de cobertura existentes, e identificando tanto sus principales impactos como las perspectivas de futuro abiertas en el diseño de una acción positiva en línea con las expectativas ciudadanas para una mayor y mejor inclusión socioterritorial en la sociedad de la información.

\section{BIBLIOGRAFÍA}

Alburquerque, F. (1997): El proceso de construcción social del territorio para el desarrollo económico local, ILPES/CEPAL, 1997.

Ballestero, F. (2002): La brecha digital: El riesgo de exclusión en la sociedad de la información. Fundación RETEVISIÓN. Madrid.

Cano, G. M., Dir. (2001): Una propuesta de comarcalización para Andalucía. Pueblos, Ciudades y Comarcas Andaluzas. Tartessos. Sevilla.

Cano, G. M., Dir. (2002): Conocer Andalucía: gran enciclopedia andaluza del siglo XXI. Tartessos, D. L. 2000-2002. Sevilla.

Casado, R., Coord. (2007): Claves de la Alfabetización Digital. ARIEL - Fundación Telefónica. [En línea] $<$ Madrid.http://www.fundacion.telefonica.com/es/debateyconocimiento/media/publi caciones/alfabetizacion_digital.pdf>

Castaño, C. y Román, C., Coords. (2002): Andalucía ante la sociedad de la información. Consejo Económico y Social de Andalucía. Sevilla.

Castells, M. (2006): La sociedad red: una visión global. Alianza Editorial. Madrid. 
Contreras, G. (2009): “Acercamiento al desarrollo tecnológico de la información y de la comunicación en la Administración Local de la Provincia de Sevilla. Municipios pequeños e intermedios". Noticias del IDR, no 5 (enero-junio). Instituto de Desarrollo Regional, Fundación Universitaria. Sevilla.

Contreras, G. (2011): "Asociacionismo en red para la planificación del desarrollo turístico sostenible. El caso del río Guadalquivir y sus principales afluentes". Revista de Estudios Andaluces, № 28, pp. 34-54.№ DOI: http:// dx.doi.org/10.12795/rea.2011.i28.03

Escalera, J. y Ruiz, E. (2006): "Asociacionismo vecinal en las ciudades medias andaluzas". Revista de Estudios Andaluces, no 26, pp. 37-66.

Esparcia, J. (2006): Políticas Públicas, Sustentabilidad y Geografía Rural. Una Agenda de Estudio (Eds.). Boletín de la Asociación de Geógrafos Españoles. Pp. 3-366.

Ferrero, F. y De la Cuesta, G. A. (2007): Libro Blanco de Buenas Prácticas para el despliegue de redes inalámbricas de banda ancha en municipios de Andalucía. Consejería de Innovación, Ciencia y Empresa (Junta de Andalucía). Sevilla.

García, I. (2005): "Políticas para la inclusión social mediante tecnologías de la información y la comunicación". Revista Electrónica Teoría de la Educación: Educación y Cultura en la Sociedad de la Información, Vol. 6, no 1, Universidad de Salamanca. Salamanca.

Gil, M. A. (1999): Dirigir y organizar en la sociedad de la información. Pirámide. Madrid.

Hernádez, M. (Coord.) (2008): Exclusión social y desigualdad. Ediciones de la Universidad de Murcia. Murcia.

Jordá, R. (1992): "Modernización tecnológica como estrategia de desarrollo para Andalucía". Revista de Estudios Andaluces, no 18, pp.27-67.

Jordá, R. y Lucendo, A. L. (2002): “Escenarios para una estadística sobre Innovación de dimensión regional. Su aplicación en Andalucía". Economía Industrial no 344, pp. 177-190. Ministerio de Industria, Turismo y Comercio. Madrid.

Norad (1997): El enfoque del marco lógico: Manual para la planificación de proyectos orientada mediante objetivos. Madrid.

Robles, J. M. (2005): La Sociedad de la Información en Andalucía. Junta de Andalucía. Sevilla.

Selwyn, N. (2004): Literature Review in Citizenship, Technology and Learning, Futurelab. Bristol.

Serrano, A. y Martínez, E. (2003): La brecha digital. Mitos y realidades. Departamento Editorial Universitaria de la Universidad Autónoma de Baja California. Baja California.

Tarroja, À. y Camagni, R. (2006): Una nueva cultura del territorio. Criterios sociales y ambientales en las políticas y el gobierno del territorio. Diputación de Barcelona. Barcelona. 
Travieso, J. L. y Planella, J. (2008): La alfabetización digital como factor de inclusión social: una mirada crítica". UOC papers, no 6, revista sobre la sociedad del conocimiento, Universidad Oberta de Catalunya. [En línea] <http://www.uoc.edu/uocpapers/6/dt/esp/travieso_planella.pdf>

Trinidad, A. (2010): Análisis y evaluación de políticas sociales. Tecnos. Madrid.

Van Winden, W. (2003): Essays on urban ICT policies. Tinbergen Institute Research Series, 328. [En línea] <http://www.euricur.nl/content_assets/WindenPDF1410.pdf>

Varone, F. y Larrue, C. (2012): Análisis y gestión de políticas públicas. Ariel. Barcelona. 